\title{
Light curing resin cements containing iodonium salts promote suitable apical bonding of posts to radicular dentin
}

Thatiana de Vicente LEITE(a) Andrea Nóbrega CAVALCANTI(b) Adriano Fonseca de LIMA(c) Luciano de Souza GONÇALVES ${ }^{(d)}$ David Christopher WATTS(e) Giselle Maria Marchi BARON(a) Luis Roberto Marcondes MARTINS ${ }^{(a)}$

(a) Universidade Estadual de

Campinas - Unicamp, Piracicaba Dental School, Department of Restorative Dentistry, Piracicaba, SP, Brazil

(b)Escola Bahiana de Medicina e Saúde Pública, Department of Restorative Dentistry, Operative Dentistry Division, Salvador, BA, Brazil.

(c) Universidade Paulista - UNIP, School of Dentistry, Dental Research Division, São Paulo, SP, Brazil.

(d) Uiversidade Federal do Rio Grande do Sul - UFRGS, Department of Restorative Dentistry, Operative Dentistry Division, Porto Alegre, RS, Brasil.

(e) University of Manchester, School of Medical Sciences and Photon Science Institute, Division of Dentistry, Manchester, UK.

Declaration of Interest: The authors certify that they have no commercial or associative interest that represents a conflict of interest in connection with the manuscript.

Corresponding Author: Gisele Maria Marchi Baron E-mail: gimarchi@fop.unicamp.br

https://doi.org/10.1590/1807-3107bor-2018.vol32.0116

Submitted: April 23, 2018

Accepted for publication: September 24, 2018 Last revision: October 02, 2018
Abstract: The aim of this study was to analyze the efficiency of experimental light-curing resin cements (ERCs) with a ternary photo-initiator system containing diphenyliodonium hexafluorphosphate (DPI) and different amines on retention of glass-fiber posts to dentin (GFP). ERCs formulations: a 1:1 mass ratio of 2,2-bis[4-(2-hydroxy-3-methacryloxypropoxy) phenylpropane and triethyleneglycol dimethacrylate. Camphorquinone was used as initiator. Six experimental groups were established according to the amine used: [ethyl-4-(dimethylamino)benzoateEDMAB or 2-(dimethylamino)ethyl methacrylate-DMAEMA] and the concentration of DPI $(0,0.5 \mathrm{~mol} \%, 1 \mathrm{~mol} \%)$. The resin cements Variolink II (dual- and light-cured versions) were used as commercial reference. Eighty recently extracted bovine incisors $(n=10)$ were selected for this study. The roots were prepared and the fiber posts were cemented with the resin cement specified for each experimental group. Specimens from coronal, middle, and apical thirds of the root were subjected to push-out bond strength test 24 hours after bonding. Data were subjected to split-plot ANOVA and the Tukey test $(p=0.05)$. ERCs containing DPI showed statistically significant higher bond strengths compared with ERCs without DPI. ERCs containing DPI were statistically similar to VARIOLINK II - dual-cured and superior to VARIOLINK II - light-cured (except for EDMAB - 1DPI in the medium third and DMAEMA - 1DPI in the coronal third). Different amines did not influence post retention. The apical root region showed the lowest bond strength for the groups EDAB-0DPI, DMAEMA-0DPI and VARIOLINK II light-cured. Light-cured ERCs containing DPI were efficient for GFP retention to radicular dentin, with similar behaviour to that of dual-curing commercial resin cement.

Keywords: Resin cements; Onium salt; Light-curing

\section{Introduction}

Pre-fabricated fiber posts have been widely used in restorative procedures for better repair of restorations after extensive coronal damage. Advantages including low cost, easy installation, and esthetic properties compared with those of metallic materials make fiber posts the first choice for the restoration of fractured teeth in many clinical situations. ${ }^{1}$ Nevertheless, their main advantage is a mechanical similarity to dentin and composites, 
which drastically reduces the risk of tooth fracture under occlusal load, and helps re-establish strength similar to that of non-prepared teeth due to better tension distribution. ${ }^{2,3,4,5}$

Because of reduced light transmission in the root canal, ${ }^{6}$ dual-cured resin cements should be used for the luting of pre-fabricated posts. The resin cements with dual-polymerization are capable of promoting suitable bonding to tooth structure; however, they also have the disadvantage of reduced working time, which can make it difficult to place the post correctly for a subsequent restoration. Glass fiber post cementation is a critical procedure with greater technical sensitivity to the operator, the development of simplified and less sensitive materials would be interesting to preserve longevity of restorations.

Optimal bond strength after cementation can be related to adequate polymerization and a high degree of conversion of the resin cement., ${ }^{7,8}$ Nevertheless, light attenuation occurs across the length of the root canal, impairing the degree of conversion in the deepest regions. ${ }^{10,11}$ To overcome this situation, some alternatives have been demonstrated to improve the polymerization of dental resin materials, such as increasing the light exposure time ${ }^{12,13}$ or the reactivity of the photo-initiator systems, ${ }^{14,15}$ improving the light-cure without extending the activation time.

Previous studies tested the addition of diphenyliodonium hexafluorphosphate (DPI) to a photo-initiator system to improve the polymerization kinetics and the degree of conversion of resin materials. ${ }^{16,17,18,19,20}$ Regarding experimental lightcuring resin cements, the presence of DPI resulted in improved mechanical properties without increased polymerization stress. ${ }^{15}$ Although this sensitizer is excited only by UV light (300 $\mathrm{nm}$ ), in the presence of another initiator, such as camphorquinone (CQ), decomposition of this salt may occur, increasing the ways of free-radical release for the polymerization of methacrylates, ${ }^{16}$ acting as a catalyst for the reaction.

The type of tertiary amine may also influence the mechanical properties of resin cements. ${ }^{20}$ Usually ethyl-4-(dimethylamino) benzoate (EDAB) or 2-(dimethylamino)ethyl methacrylate (DMAEMA) is used, combined with CQ as a reducing agent. Both have different chemical properties: EDAB has an aromatic ring and a higher ability to donate electrons compared with DMAEMA, which presents an aliphatic structure and hydrophilic behavior.

As demonstrated in a previous study, ${ }_{1}^{15}$ DPI may lead light-curing resin cements to better polymerization. Nevertheless, it is unknown if these better results can improve the efficacy of this agent in clinical procedures when reduced irradiance reaches the resin materials (e.g. middle and apical regions of the root canal). Previous study evaluated the effect of DPI on bond strength of fiber posts. ${ }^{21}$ However, the study evaluated different concentration of DPI using only one amine in the initiation system, without any commercial reference to compare the efficiency of the experimental cements with the current ones. Therefore, the aim of this study was to investigate the influence of different DPI concentrations combined with two tertiary amines on the bond strength of glass-fiber posts fixed with an experimental light-curing resin cement (ERC), comparing the bonding efficacy with that of commercial light-curing and dual resin cements under different regions of the root canal. The hypotheses tested were: a three-component photo-initiator system containing an onium salt and different tertiary amines influence the regional bond strength of experimental photo-activated resin cements.

\section{Methodology}

\section{Formulation of the experimental resin cements}

A model blend based on a 1:1 ratio (wt) of 2,2-bis[4(2-hydroxy-3-methacryloxypropoxy) phenyl]propane (Bis-GMA) and triethyleneglycol dimethacrylate (TEGDMA) (Esstech Inc., Essington, USA) was prepared with $1 \mathrm{~mol} \%$ of camphorquinone (CQ-Esstech Inc.) as initiator. The experimental groups were established according to the amine used ( $2 \mathrm{~mol} \%$ of DMAEMA or $2 \mathrm{~mol} \%$ of EDAB, both from Sigma-Aldrich, St. Louis, USA) or the concentration of DPI (Sigma-Aldrich, St. Louis, USA) used (0, 0.5, or $1 \mathrm{~mol} \%)$. Butylated hydroxytoluene (BHT, Sigma-Aldrich, St. Louis, USA) $0,1 \mathrm{~mol} \%$ was added as inhibitor. Each formulation was loaded with $60 \%$ by weight of silanated barium borosilicate glass fillers (Esstech, $0.7 \mu \mathrm{m}$ average 
size). The chemicals were used without further purification. The manipulation of the ERC was carried out in an environment with controlled humidity and temperature, under yellow fluorescent light.

\section{Specimen preparation}

Eighty recently extracted bovine incisors with similar root sizes and lengths were selected for this study. The crowns were removed $1 \mathrm{~mm}$ below the cemento-enamel junction (CEJ) by means of a water-cooled low-speed diamond saw (Isomet; Buehler, Lake Bluff, USA) to obtain 15-mm root segments. Roots with opened apices and/or a curvature greater than $10^{\circ}$ were not included in the study. The post space was prepared with a Largo drill \#4 (Dentsply Maillefer, Ballaigues, Switzerland) for the cementation of glass-fiber post \#3 (1.5 mm diameter, Reforpost Ângelus Produtos Odontológicos, Londrina, Brazil).

Table 1. Bond strength means (standard deviations) of experimental groups according to the different root canal regions.

\begin{tabular}{lccc}
\hline \multirow{2}{*}{ Resin cements } & \multicolumn{3}{c}{ Dentin root region } \\
\cline { 2 - 4 } & \multicolumn{1}{c}{ Coronal } & \multicolumn{1}{c}{ Middle } & Apical \\
\hline EDAB-0mol\% DPI & $9.4(2.9)^{\mathrm{Da}}$ & $6.2(2.3)^{\mathrm{CDab}}$ & $4.7(2.7)^{\mathrm{BCb}}$ \\
EDAB-0.5mol\% DPI & $23.3(7.3)^{\mathrm{Aa}}$ & $16.0(4.8)^{\mathrm{Ab}}$ & $6.6(3.0)^{\mathrm{ABCc}}$ \\
EDAB-1 mol\% DPI & $20.6(3.3)^{\mathrm{ABa}} 10.0(1.8)^{\mathrm{BCb}} 9.54(2.7)^{\mathrm{ABb}}$ \\
DMAEMA-Omol\% DPI & $13.1(2.6)^{\mathrm{CDa}} 3.7(1.2)^{\mathrm{Db}}$ & $3.9(2.1)^{\mathrm{Cb}}$ \\
DMAEMA-0.5mol\% DPI & $20.4(6.5)^{\mathrm{ABa}} 14.4(5.8)^{\mathrm{ABb}}$ & $9.9(4.1)^{\mathrm{ABc}}$ \\
DMAEMA-1 mol\% DPI & $17.8(5.5)^{\mathrm{BCa}} 12.6(3.5)^{\mathrm{ABb}}$ & $9.9(3.9)^{\mathrm{Ab}}$ \\
VARIOLINK II Ligth-Cured & $17.1(6.0)^{\mathrm{BCa}} 9.8(4.1)^{\mathrm{BCb}}$ & $3.6(2.1)^{\mathrm{Cc}}$ \\
VARIOLINK II DUAL & $20.4(8.0)^{\mathrm{ABa}} 17.5(3.8)^{\mathrm{Aa}} 7.7(3.7)^{\mathrm{ABCb}}$ \\
\hline
\end{tabular}

The roots were filled with gutta-percha (Dentsply Indústria e Comércio ltda, Petrópolis, Brazil) using the lateral condensation technique. Each root was then stored in distilled water $(1.5 \mathrm{~mL})$ for 24 hours at $37^{\circ} \mathrm{C}$. Thereafter, the gutta-percha was removed with Largo drill \#5 (Dentsply Maillefer, Ballaigues, Switzerland), and the post space was prepared to depths of $12 \mathrm{~mm}$ from the cervical margin, leaving an apical seal of $3 \mathrm{~mm}$. The roots were randomly divided into eight groups $(n=10)$ according to the experimental formulation determined by the photoinitiator system used (Table 1).

Distinct uppercase letters indicate statistically significant differences between resin cements in the same column; and distinct lowercase letters indicate statistically significant differences between the dentin root regions in the same row . Split-plot ANOVA and Tukey's test ( $\mathrm{p}<0.05)$.

A description of the composition of commercial materials used in this study is shown in Table 2.

\section{Post cementation}

The adhesive protocol used for all groups was as follows: After acid-etching with 35\% phosphoric acid for $15 \mathrm{~s}$ (Scotchbond Etchant Gel, 3M ESPE, St. Paul, MN, USA), the root canal was washed with distilled water for $15 \mathrm{~s}$, and the excess of water was removed with absorbent paperpoints (Dentsply Maillefer, Ballaigues, Suisse). The adhesive system (Adper Scotchbond Multipurpose, 3M ESPE, St. Paul, USA) was then applied following the manufacturer's instructions: primer agent, followed by a 5 -second gentle air-spray, and then the hydrophobic layer of

Table 2. Commercial materials used in this study.

\begin{tabular}{|c|c|c|}
\hline Material & Manufacture & Chemical composition \\
\hline Scotchbond Etchant gel & 3M ESPE - St. Paul, MN, USA & Water, phosphoric acid, poly(vinyl alcohol) \\
\hline \multirow[t]{2}{*}{ Adper scotchbond multipurpose } & \multirow[t]{2}{*}{ 3M ESPE - St. Paul, MN, USA } & $\begin{array}{c}\text { Primer - water, 2-hydroxyethyl metacrylate (HEMA), copolymer of } \\
\text { acrylic and itaconic acids }\end{array}$ \\
\hline & & Adhesive - bisphenol A diglycidyl ether dimethacrylate (BISGMA) \\
\hline Variolink II & $\begin{array}{l}\text { Ivoclar Vivadent AG - Schaan, } \\
\text { Liechtenstein }\end{array}$ & $\begin{array}{l}\text { The monomer matrix is composed of Bis-GMA, urethane } \\
\text { dimethacrylate (UDMA), and triethylene glycol dimethacrylate } \\
\text { (TEGDMA). The inorganic fillers are barium glass, ytterbium } \\
\text { trifluoride, Ba-Al-fluorosilicate glass, and spheroid mixed oxide. } \\
\text { Additional contents: catalysts, stabilizers, and pigments. The particle } \\
\text { size is } 0.04-3.0 \mu \mathrm{m} \text {. The mean particle size is } 0.7 \mu \mathrm{m}\end{array}$ \\
\hline Ceramic primer & 3M ESPE - St. Paul, MN, USA & Ehtyl acohol, water, methacryloxymetpropyltrimetoxisilane \\
\hline
\end{tabular}


adhesive agent. The adhesive was light-cured for $20 \mathrm{~s}$ with a light-emitting diode (LED-Bluephase G2, Ivoclar Vivadent, Schaan, Liechtenstein) with irradiance $1200 \mathrm{~mW} / \mathrm{cm}^{2}$.

The experimental resin cement and the commercial reference were inserted into the root canal by means of a syringe (Centrix, Inc., 770 River Road, Shelton, CT, USA) to fill the entire root canal. The commercial resin cement (Variolink II, Ivoclar Vivadent, Schaan, Liechtenstein) was used as a commercial reference in light-cured (base paste) and dual-cured (base paste and catalyst paste) modes, prepared following the manufacturer's instructions.

The fiber post was pre-treated with $35 \%$ phosphoric acid (Scotchbond Etchant Gel, 3M ESPE, St. Paul, USA), washed, and dried, and then a silane layer was applied (Ceramic Primer, 3M ESPE, St. Paul, USA). After this, the post was inserted into the root canal filled with the resin cement established for each group, and excess luting material was removed. The cements (experimental and commercial reference) were light-cured continuously for $60 \mathrm{~s}$ from the top of the glass fiber post, directed perpendicular to the root, by means of the previously mentioned LED source. After cementation, each root was kept in the dark, maintained in relative humidity at $37^{\circ} \mathrm{C}$.

Twenty-four hours after the cementation, the roots were sectioned perpendicular to their long axis in three slices ( $1 \mathrm{~mm}$ thick), using a low-speed diamond saw (Isomet 1000, Buehler) with constant water coolant. The first slice was discarded to obtain standardized and smooth samples. The samples were divided in accordance with the region ( $1 \mathrm{~mm}$ - coronal, $5 \mathrm{~mm}$ - middle, and $9 \mathrm{~mm}$ - apical).

\section{Push-out bond strength test}

For the push-out test, the slices were fixed in a base with a central hole $(3 \mathrm{~mm})$ in a load-testing machine (EMIC DL 500, São José dos Pinhais, Brazil) and stressed to failure at a crosshead speed of 0.5 $\mathrm{mm} / \mathrm{min}$. The data obtained in $\mathrm{kgf}$ were converted to $\mathrm{MPa}$, as described in a previous study. ${ }^{22}$

\section{Failure analysis}

The failure mode was evaluated by stereomicroscopy (×50, Meiji 2000, Meiji Techno, Saitama, Japan) at ×50 magnification. Fracture mode categories were classified into three groups: AP - adhesive between fiber post and luting agent; $\mathrm{AD}$ - adhesive between dentin and resin cement; and $\mathrm{M}$ - mixed failure.

\section{Statistical analysis}

Data obtained from push-out bond strength tests (MPa) were subjected to split-plot ANOVA two-way (resin cement and root region) after analysis of the normal distribution of data and homoscedasticity of variances. Multiple comparisons were performed by the Tukey post-hoc test. The level of significance was set at 5\% (SAS Institute Inc., Cary, USA).

\section{Results}

\section{Push-out bond-strength test}

The interaction between the factors "resin cement" and "root region" was significant ( $p<0.001)$. The push-out bond strength decreased with the increase of the root canal depth (Table 1), but in some instances the apical region was similar to medium third, as observed in the groups EDAB - 1mol\% DPI, DMAEMA 0 and $1 \mathrm{~mol} \%$ DPI. In DPI-containing cements, the presence of salt $(0.5 \mathrm{~mol} \%)$ was capable of significantly increasing the push-out bond strength of fiber-posts compared with ERCs without DPI, excepting the apical region for EDAB-containing cements. The cements with DPI also presented results statistically similar to those of the commercial reference Variolink II dual-cured, except for groups containing DMAEMA $1 \mathrm{~mol} \% \mathrm{DPI}$ in the coronal region and EDAB - $1 \mathrm{~mol} \%$ DPI in the middle region, which were comparable with Variolink II - light-cured. In the apical region, the commercial reference Variolink II - light-cured mode presented the lowest results, similar to groups EDAB and DMAEMA without DPI.

\section{Fracture mode analysis}

It was noted that the most prevalent failure occurred in the adhesive layer between dentin and resin cement (AD) (Figure). Fractures occurred on the adhesive layer between post and cement, and mixed failures were also observed. However, the frequency of this kind of failure was significantly lower than the AD mode. 


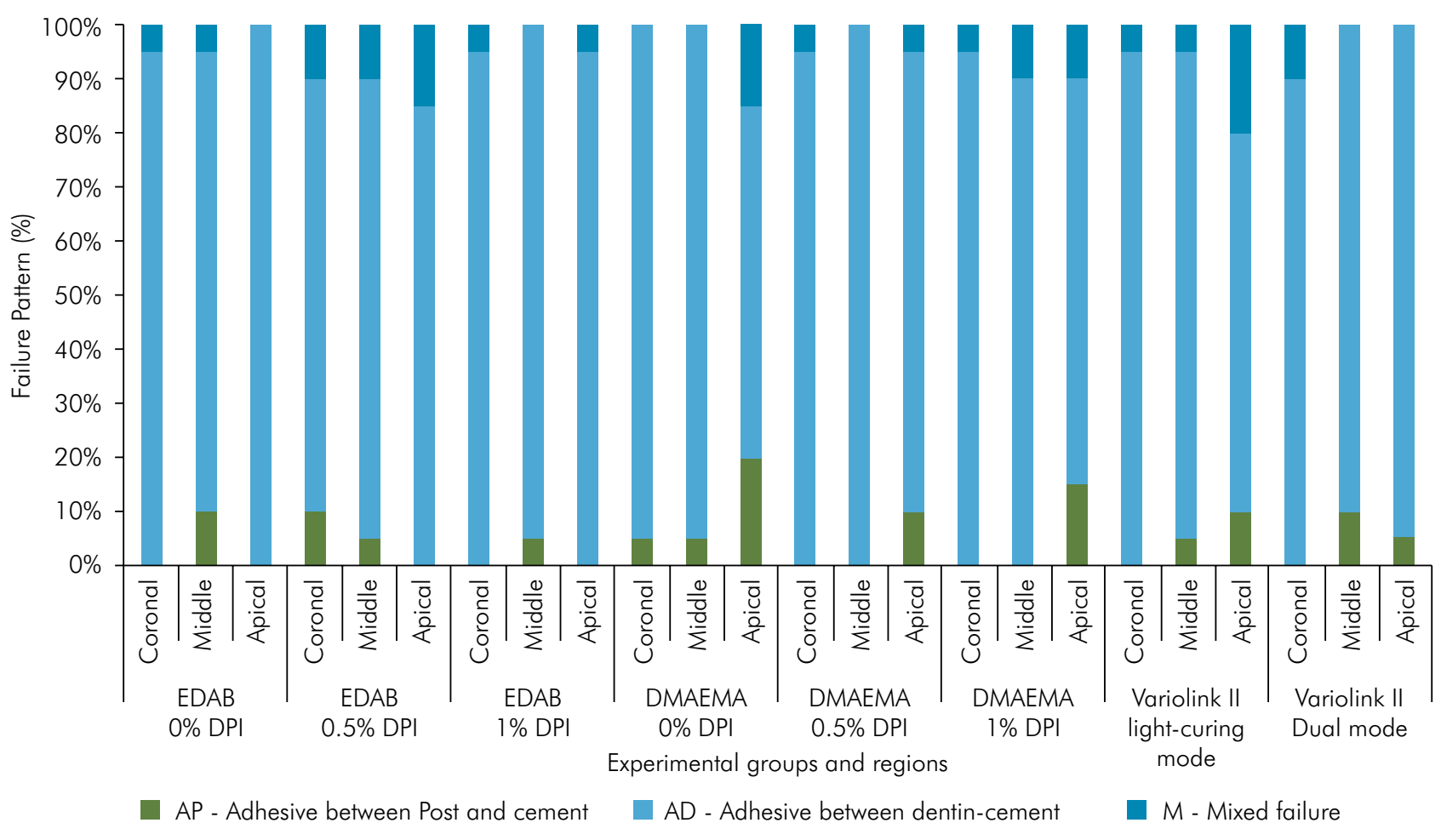

Figure. Distribution of the failure mode according to the experimental group and region evaluated.

\section{Discussion}

The first hypothesis of the present study, that a three-component initiator system containing DPI and different tertiary amines influence the regional bond strength of experimental photo-activated resin cements, was not accepted. Although the bond strength of ERCs containing $0.5 \mathrm{~mol} \%$ DPI was significantly increased compared with that of ERCs without iodonium salt, regardless of the root canal region, there was no statistical difference between EDAB - 0mol\% DPI and EDAB - 1mol\% DPI at the middle and apical thirds, as well between DMAEMA - 0 mol\% DPI and DMAEMA - $1 \mathrm{~mol} \%$ DPI at the coronal third. The potential of DPI salt to promote better polymerization and mechanical properties has been demonstrated in previous studies. ${ }^{15,16,23}$ Although DPI cannot be activated by blue light, it reacts with excited $C Q$, producing a triplet state, and an active phenyl radical is generated, which is effective in initiating the polymerization reaction. Another mechanism involving the salt is that the free radicals produced by the interaction between CQ and the amine can also react with DPI, breaking the C-I bond and releasing another reactive phenyl radical, which can further react with residual amines, abstracting a proton and generating a new amine free radical. As a consequence of this improved polymerization, the ERCs containing DPI were able to achieve bond strength similar to that of the dual-cured commercial reference tested, and superior to that of the light-cured commercial reference. This better performance of DPIcontaining cements can be explained based on the better polymerization, which promotes polymers with mechanical properties superior to those of cements without DPI, as demonstrated previously. ${ }^{15}$

However, the groups containing $1 \mathrm{~mol} \%$ of DPI had intermediary bond strength, with both DMAEMA and EDAB. The increased concentration of DPI in these cements can probably promote a greater amount of free radicals, promoting premature termination, reducing the physical properties of the polymer, as shown in a previous study. ${ }^{15}$ We also speculate that the higher polymerization stress of the ERCs containing $1 \mathrm{~mol} \%{ }^{15}$ can influence the bond strength to dentin, which, allied 
to the high C-factor of the root canal, ${ }^{24}$ can be responsible for the intermediary values obtained. Therefore, based on the present results, the best concentration to obtain the highest bond strength of fiber posts to the root canal is $0.5 \mathrm{~mol} \%$. As demonstrated in a previous study, high concentrations of DPI ( 1 and $2 \mathrm{~mol} \%$ ) can reduce the mechanical properties and increase the polymerization stress of the cement. These characteristics, allied with the intermediate results of the effects of cements containing $1 \mathrm{~mol} \%$ of DMAEMA and EDAB on bond strength, observed in the present study, highlight the ideal concentration as $0.5 \mathrm{~mol} \%$ of DPI.

The impaired performance of glass-fiber posts subjected to push-out bond strength tests in the apical region has been well-documented, ${ }^{25,26}$ corroborating the findings of the present study, due to the reduced irradiation reaching the resin cement in this region. This negative effect was more evident in groups using cements without DPI and in the light-cured commercial reference. Moreover, many adverse factors in this region should be considered, such as reduced density of dentin tubules, ${ }^{27}$ highest $\mathrm{C}$-factor, and, consequently, increased stress of polymerization. ${ }^{24}$ However, it should be highlighted that the presence of DPI improved the bond strength in the apical region, equalling, in some cases, the results in the middle region and better than the results in groups using cements without DPI and in the light-cured commercial reference.

Previous study evaluated the effect of push-out bond strength of resin cements containing DPI to root canal dentin, showing contrasting results. ${ }^{28}$ Despite the higher polymerization stress presented by the cement without DPI, the previous study demonstrated better results for this cement in the cervical region. ${ }^{28}$ In the present study, the experimental cements without DPI had lower bond strength compared to the cements containing the salt, probably due to the better polymerization reaction. In addition, the present study evaluated two different amines in the initiator system, comparing with light- and dual polymerization commercial references, demonstrating the efficacy and promisor results of DPI cements to bond fiber posts to dentin. Despite the molecular structural differences both amines behaved similarly in the different root regions.

Satisfactory adhesion to radicular dentin is still a challenge; therefore, the selection of the adhesive system is an important step in glass-fiber post cementation. In this study the protocol was evaluated with a threestep light-curing adhesive system so that there was no negative interaction between acid monomers present in superficial layer of two-step etch and rinse adhesive systems with tertiary amines of the dualcured commercial reference Variolink II. The use of dual adhesive systems was discarded since the components would possibly influence resin cement polymerization, modulating the results of the different formulations and jeopardizing the analysis of resin cement composition. In analysis of the failure mode, it can be noted that the higher frequency of the AD mode for all groups may indicate that the hybrid layer was the weakest link of the adhesion. These results probably occurred due to the difficult curing of the dental adhesive. Nevertheless, when we observed the results and distinct behavior of the cements, even with these characteristics of the bonding interface, the comparison between the different formulations was not compromised.

Dual-cured resin cement used as commercial reference attained the highest result for bond strength to root canal dentin. However, the light-curing resin cements containing DPI had statistically similar results to the dual-cure resin, clearly indicating the efficiency of ternary photo-initiator systems containing DPI for optimization of the polymerization reaction of lightcured resin cements, even in deep regions. The use of light-curing resin cements containing DPI can allow for reliable bonding with better working time compared with the dual-cure resin cements, indicating their promise as materials for the repair of posts and ceramic restorations, even in situations with reduced irradiation.

\section{Conclusions}

Retention of glass-fiber posts luted with experimental light-cured resin cement containing DPI was similar to that of those fixed with the dualcured commercial reference.

The concentration of $0.5 \mathrm{~mol} \%$ of DPI allowed for the best bond strength in most conditions tested.

Different tertiary amines may not cause different bond strength values in the light protocol tested.

The apical region presented the lowest bond strength values. 


\section{Acknowledgments}

The authors deny any conflicts of interest in this study. This study was supported by CAPES (Coordination for the Improvement of Higher Education Personnel).

\section{References}

1. Christensen GJ. Posts and cores: state of the art. J Am Dent Assoc. 1998 Jan;129(1):96-7. https://doi.org/10.14219/jada.archive.1998.0028

2. Schwartz RS, Robbins JW. Post placement and restoration of endodontically treated teeth: a literature review. J Endod. 2004 May;30(5):289-301. https://doi.org/10.1097/00004770-200405000-00001

3. Bitter K, Kielbassa AM. Post-endodontic restorations with adhesively luted fiber-reinforced composite post systems: a review. Am J Dent. 2007 Dec;20(6):353-60.

4. Soares PV, Santos-Filho PC, Martins LR, Soares CJ. Influence of restorative technique on the biomechanical behavior of endodontically treated maxillary premolars. Part I: fracture resistance and fracture mode. J Prosthet Dent. 2008 Jan;99(1):307. https://doi.org/10.1016/S0022-3913(08)60006-2

5. Figueiredo FE, Martins-Filho PR, Faria-E-Silva AL. Do metal post-retained restorations result in more root fractures than fiber post-retained restorations? A systematic review and meta-analysis. J Endod. 2015 Mar;41(3):309-16. https://doi.org/10.1016/i.joen.2014.10.006

6. Faria e Silva AL, Arias VG, Soares LE, Martin AA, Martins LR. Influence of fiber-post translucency on the degree of conversion of a dual-cured resin cement. J Endod. 2007 Mar;33(3):303-5. https://doi.org/10.1016/i.joen.2006.11.015

7. Asmussen E, Peutzfeldt A. Resin composites: strength of the bond to dentin versus surface energy parameters. Dent Mater. 2005 Nov;21(11):1039-43. https://doi.org/10.1016/i.dental.2004.12.004

8. Kanehira M, Finger WJ, Hoffmann M, Endo T, Komatsu M. Relationship between degree of polymerization and enamel bonding strength with self-etching adhesives. J Adhes Dent. 2006 Aug;8(4):211-6.

9. Khabeer A, Whitworth J, Rolland S. Polymerization kinetics of resin cements after light activation through fibre posts: an in vitro study. Int Endod J. 2015 Mar;48(3):261-7. https://doi.org/10.1111/iej.12309

10. Foxton RM, Nakajima M, Tagami J, Miura H. Bonding of photo and dual-cure adhesives to root canal dentin. Oper Dent. 2003 Sep-Oct;28(5):543-51.

11. Dogar A, Altintas SC, Kavlak S, Guner A. Determining the influence of fibre post light transmission on polymerization depth and viscoelastic behaviour of dual-cured resin
The authors are grateful to Esstech Inc., Essington, PA, USA for a generous donation of some materials used in the present study.

cement. Int Endod J. 2012 Dec;45(12):1135-40.

https://doi.org/10.1111/j.1365-2591.2012.02085.x

12. Halvorson RH, Erickson RL, Davidson CL.

Energy dependent polymerization of resin-based

composite. Dent Mater. 2002 Sep;18(6):463-9.

https://doi.org/10.1016/S0109-5641(01)00069-0

13. Moraes RR, Faria-e-Silva AL, Ogliari FA, Correr-Sobrinho L, Demarco FF, Piva E. Impact of immediate and delayed light activation on self-polymerization of dual-cured dental resin luting agents. Acta Biomater. 2009 Jul;5(6):2095-100. https://doi.org/10.1016/i.actbio.2009.01.030

14. Brandt WC, Schneider LF, Frollini E, Correr-Sobrinho $L$, Sinhoreti MA. Effect of different photo-initiators and light curing units on degree of conversion of composites. Braz Oral Res. 2010 Jul-Sep;24(3):263-70. https://doi.org/10.1590/S1806-83242010000300002

15. Gonçalves LS, Moraes RR, Ogliari FA, Boaro L, Braga RR, Consani S. Improved polymerization efficiency of methacrylate-based cements containing an iodonium salt. Dent Mater. 2013 Dec;29(12):1251-5. https://doi.org/10.1016/i.dental.2013.09.010

16. Ogliari FA, Ely C, Petzhold CL, Demarco FF, Piva E. Onium salt improves the polymerization kinetics in an experimental dental adhesive resin. J Dent. 2007 Jul;35(7):583-7. https://doi.org/10.1016/i.jdent.2007.04.001

17. Guo X, Wang Y, Spencer P, Ye Q, Yao X. Effects of water content and initiator composition on photopolymerization of a model BisGMA/HEMA resin. Dent Mater. 2008 Jun;24(6):82431. https://doi.org/10.1016/i.dental.2007.10.003

18. Andrade KM, Palialol AR, Lancellotti AC, Aguiar FH, Watts DC, Gonçalves LS et al. Effect of diphenyliodonium hexafluorphosphate on resin cements containing different concentrations of ethyl 4-(dimethylamino)benzoate and 2-(dimethylamino)ethyl methacrylate as co-initiators. Dent Mater. 2016 Jun;32(6):749-55. https://doi.org/10.1016/i.dental.2016.03.014

19. Dressano D, Palialol AR, Xavier TA, Braga RR, Oxman JD, Watts DC et al. Effect of diphenyliodonium hexafluorophosphate on the physical and chemical properties of ethanolic solvated resins containing camphorquinone and 1-phenyl-1,2-propanedione sensitizers as initiators. Dent Mater. 2016 Jun;32(6):756-64. https://doi.org/10.1016/i.dental.2016.03.010 
Light curing resin cements containing iodonium salts promote suitable apical bonding of posts to radicular dentin

20. Schroeder WF, Vallo Cl. Effect of different photoinitiator systems on conversion profiles of a model unfilled lightcured resin. Dent Mater. 2007 Oct;23(10):1313-21. https://doi.org/10.1016/j.dental.2006.11.010

21. Augusto CR, Leitune VC, Ogliari FA, Collares FM. Influence of an iodonium salt on the properties of dual-polymerizing selfadhesive resin cements. J Prosthet Dent. 2017 Aug;118(2):22834. https://doi.org/10.1016/i.prosdent.2016.10.013

22. Goracci C, Tavares AU, Fabianelli A, Monticelli F, Raffaelli O, Cardoso PC et al. The adhesion between fiber posts and root canal walls: comparison between microtensile and push-out bond strength measurements. Eur J Oral Sci. 2004 Aug;112(4):353-61. https://doi.org/10.1111/j.1600-0722.2004.00146.x

23. Kukiattrakoon B, Thammasitboon K. The effect of different etching times of acidulated phosphate fluoride gel on the shear bond strength of high-leucite ceramics bonded to composite resin. J Prosthet Dent. 2007 Jul;98(1):17-23. https://doi.org/10.1016/S0022-3913(07)60033-X

24. Garcia FC, Otsuki M, Pashley DH, Tay FR, Carvalho RM. Effects of solvents on the early stage stiffening rate of demineralized dentin matrix. J Dent. 2005 May;33(5):371-7. https://doi.org/10.1016/i.jdent.2004.10.013

25. Farina AP, Cecchin D, Garcia LF, Naves LZ, Correr Sobrinho $L$, Pires-de-Souza FC. Bond strength of fiber posts in different root thirds using resin cement. J Adhes Dent. 2011 Apr;13(2):179-86. https://doi.org/10.3290/i.jad.a18444

26. Machado FW, Bossardi M, Ramos TS, Valente LL, Münchow EA, Piva E. Application of resin adhesive on the surface of a silanized glass fiber-reinforced post and its effect on the retention to root dentin. J Endod. 2015 Jan;41(1):106-10. https://doi.org/10.1016/i.joen.2014.09.014

27. Ferrari M, Mannocci F, Vichi A, Cagidiaco MC, Miör IA. Bonding to root canal: structural characteristics of the substrate. Am J Dent. 2000 Oct;13(5):255-60.

28. Lopes MB, Santos AM, Coelho D, Gonini A, Ogliari FA, Moraes RR. Influence of diphenyliodonium hexafluorophosphate on the bond strength and mechanical properties of model resin cements. Int J Adhes Adhes. 2013;47:125-8. https://doi.org/10.1016/i.ijadhadh.2013.08.006 